Acta Crystallographica Section D

Biological

Crystallography

ISSN 0907-4449

\section{Robert T. Byrne, ${ }^{a} \neq$ Fiona Whelan, ${ }^{a} \S$ Pierre Aller, ${ }^{b}$ Louise E. Bird, ${ }^{c, d}$ Adam Dowle, Carina M. C. Lobley, ${ }^{b}$ Yamini Reddivari, ${ }^{c, d}$ Joanne $E$. Nettleship, ${ }^{\text {,d d }}$ Raymond J. Owens, ${ }^{\text {c,d }}$ Alfred A. Antson ${ }^{\mathrm{a}}$ and David G. Waterman ${ }^{\mathfrak{f}}$ *}

${ }^{a}$ York Structural Biology Laboratory, Department of Chemistry, University of York, Heslington YO10 5DD, England, ${ }^{\mathbf{b}}$ Diamond Light Source Ltd, Diamond House, Harwell Science and Innovation Campus, Didcot, Oxfordshire OX11 ODE, England, 'OPPF-UK, Research Complex at Harwell, R92 Rutherford Appleton Laboratory, Didcot, Oxfordshire OX11 OFA, England, dDivision of Structural Biology, Oxford University, Wellcome Trust Centre for Human Genetics, Roosevelt Drive, Oxford OX3 7BN, England, ${ }^{\mathbf{e}}$ Bioscience Technology Facility, Department of Biology, University of York, Heslington YO10 5DD, England, and 'STFC, Rutherford Appleton Laboratory, Didcot, Oxfordshire OX11 OFA, England

₹ Current address: Section of Structural Biology, The Institute of Cancer Research, Chester Beatty Laboratories, 237 Fulham Road, London SW3 6JB, England.

$\S$ Current address: Department of Biology, University of York, Heslington YO10 5DD England.

Correspondence e-mail: david.waterman@stfc.ac.uk

\title{
S-Adenosyl-S-carboxymethyl-L-homocysteine: a novel cofactor found in the putative tRNA-modifying enzyme CmoA
}

Uridine at position 34 of bacterial transfer RNAs is commonly modified to uridine-5-oxyacetic acid $\left(\mathrm{cmo}^{5} \mathrm{U}\right)$ to increase the decoding capacity. The protein $\mathrm{CmoA}$ is involved in the formation of $\mathrm{cmo}^{5} \mathrm{U}$ and was annotated as an $S$-adenosyl-Lmethionine-dependent (SAM-dependent) methyltransferase on the basis of its sequence homology to other SAMcontaining enzymes. However, both the crystal structure of Escherichia coli CmoA at $1.73 \AA$ resolution and mass spectrometry demonstrate that it contains a novel cofactor, $S$-adenosyl- $S$-carboxymethyl-L-homocysteine (SCM-SAH), in which the donor methyl group is substituted by a carboxymethyl group. The carboxyl moiety forms a salt-bridge interaction with Arg199 that is conserved in a large group of CmoA-related proteins but is not conserved in other SAMcontaining enzymes. This raises the possibility that a number of enzymes that have previously been annotated as SAMdependent are in fact SCM-SAH-dependent. Indeed, inspection of electron density for one such enzyme with known X-ray structure, PDB entry $1 \mathrm{im} 8$, suggests that the active site contains SCM-SAH and not SAM.

\section{Introduction}

Following transcription by RNA polymerase, a transfer RNA transcript is converted into a mature tRNA through processing and nucleoside modification. Processing in bacteria entails the removal of the $5^{\prime}$ leader and $3^{\prime}$ tail sequences and, if necessary, the re-synthesis of the $3^{\prime}$ CCA extension required for aminoacylation. Nucleoside modification is part of the maturation process that extends the physicochemical properties of tRNA by providing a wider complement of nucleosides than the canonical four introduced by RNA polymerase during transcription. Approximately $10 \%$ of nucleosides in a typical tRNA are modified, which corresponds to around seven modified nucleosides per tRNA (Jühling et al., 2009). Of the 109 modified nucleosides of RNA, 93 are found in tRNA, making it the most diversely modified RNA (Cantara et al., 2011). Modified nucleosides confer diverse properties upon tRNA, but they generally fine-tune the structure and stability such that it is optimized for functioning in processes such as aminoacylation and translation (Motorin \& Helm, 2010).

The anticodon stem loop (ASL) is one of the most heavily modified regions of tRNA and is frequently modified at positions 34 (the wobble position) and 37 (immediately after the anticodon) (Jühling et al., 2009). Modification of the ASL reduces its flexibility and shifts its structure towards the U-turn conformation; this has been seen in crystal structures of mature tRNA, solution structures of isolated modified ASL domains and structures of the $70 \mathrm{~S}$ ribosome in complex with tRNA and mRNA (Selmer et al., 2006; Shi \& Moore, 2000;
Received 26 January 2013 Accepted 20 February 2013

PDB Reference: $\mathrm{CmoA}$, 4iwn 
Vendeix et al., 2008). Because modifications impose this conformation upon the ASL, the entropic penalty otherwise associated with remodelling during translation is avoided (Agris, 2008). A number of nucleoside modifications at positions 34 and 37 also expand the decoding capacity beyond that predicted by the 'Wobble hypothesis' (Agris et al., 2007). In bacteria, the 5-oxyuridine derivatives commonly found at position 34 fall into this category because they allow the tRNA to decode not only codons ending in A and $\mathrm{G}$ (as predicted by the Wobble hypothesis), but also $\mathrm{U}$ and $\mathrm{C}$, so that a single tRNA can consequently decode all four codons within a single box of the genetic code (Nasvall et al., 2004). The derivative uridine-5-oxyacetic acid $\left(\mathrm{cmo}^{5} \mathrm{U}\right)$ has so far been found in tRNA ${ }^{\text {Ala }}$, $\mathrm{tRNA}^{\text {Pro }}$, $\mathrm{tRNA}^{\mathrm{Ser}}$ and tRNA ${ }^{\mathrm{Val}}$ (Jühling et al., 2009). These modifications are therefore beneficial because they not only reduce the entropic penalty associated with translation but also reduce the number of different tRNAs that a cell must produce for decoding.

While the function of $\mathrm{cmo}^{5} \mathrm{U}$ within the context of a tRNA is well characterized, its synthesis has received relatively little attention. The proposed biosynthetic pathway involves the stepwise modification of $\mathrm{U}$ to $\mathrm{cmo}^{5} \mathrm{U}$ through the intermediates 5-hydroxyuridine (ho $\left.{ }^{5} \mathrm{U}\right)$ and 5-methoxyuridine $\left(\mathrm{mo}^{5} \mathrm{U}\right)$ (Fig. 1). In keeping with the majority of other nucleoside-modification pathways, it is likely that the substrate base is modified while remaining part of the tRNA and is not simply exchanged for a pre-modified base in a transglycosylation reaction. Mutations in the $\mathrm{cmoB}$ and $\mathrm{cmo} A$ genes result in accumulation of $\mathrm{ho}^{5} \mathrm{U}$ and $\mathrm{mo}^{5} \mathrm{U}$, respectively, indicating that $\mathrm{CmoB}$ is involved in the modification of $\mathrm{ho}^{5} \mathrm{U}$ to $\mathrm{mo}^{5} \mathrm{U}$ and that $\mathrm{CmoA}$ is involved in the modification of $\mathrm{mo}^{5} \mathrm{U}$ to $\mathrm{cmo}^{5} \mathrm{U}$ (Nasvall et al., 2004). Both $\mathrm{CmoA}$ and $\mathrm{CmoB}$ contain $S$-adenosylmethionine (SAM) binding motifs, hinting that they are methyltransferases, but only one of the two $\mathrm{C}$ atoms in the side chain of $\mathrm{cmo}^{5} \mathrm{U}$ is derived from SAM (Hagervall et al., 1990). Furthermore, the synthesis of 5-oxyuridine derivatives is also dependent upon chorismic acid, although the nature of this dependency has not yet been determined (Hagervall et al., 1990; Nasvall et al., 2004). This suggests that the modification pathway has not been fully elucidated or that parts of the $\mathrm{cmo}^{5} \mathrm{U}$ side chain may be derived from other metabolites.

We decided to investigate the functions of $\mathrm{CmoA}$ and $\mathrm{CmoB}$ in detail by $\mathrm{X}$-ray crystallography to help fill the gaps in our understanding of $\mathrm{cmo}^{5} \mathrm{U}$ biosynthesis. Although $\mathrm{CmoA}$ was expected to be similar to the known structure of its homologue Haemophilus influenzae $\mathrm{YecO}$, there is no sufficiently high-quality model of $\mathrm{CmoB}$ in the Protein Data Bank to form structurebased hypotheses about the function of the system. We hope to rectify this by

Figure 1 identified. providing high-quality structures of both proteins from the same target organism, allowing detailed models of the enzymatic pathway and RNA interactions to be constructed and further tested. Here, we report the structure of Escherichia coli $\mathrm{CmoA}$, which unexpectedly reveals a cofactor that, to the best of our knowledge, has not been observed before.

\section{Materials and methods}

\subsection{Molecular biology and protein production}

The coding sequence $c m o A$ was amplified from OmniMax II cells (Invitrogen) and cloned into the vector pOPINF using the In-Fusion method to generate the construct OPPF7299 (Berrow et al., 2007). The final construct has an $\mathrm{R} 100 \mathrm{H}$ point mutation with respect to the deposited sequence of $\mathrm{CmoA}$ from E. coli K-12 strain MG1655 (UniProt P76290), which may be either a PCR mutation or a genuine difference in this strain. Sequence analysis shows that amino acids with diverse properties are found at this position in other UniProt CmoAfamily members. Once the X-ray structure had been determined, it became clear that this residue is located on the surface of the protein on the side opposite to the dimer interface and at a distance of $\sim 15 \AA$ from the nearest atom of the SCM-SAH cofactor. E. coli Rosetta pLysS (DE3) cells were transformed with the resulting vector and grown in Overnight Express Instant TB medium (Merck). The cells were incubated at $310 \mathrm{~K}$ until an $\mathrm{OD}_{600 \mathrm{~nm}}$ of 0.6 was attained, at which point the temperature was reduced to $298 \mathrm{~K}$ and the cells were grown for a further $20 \mathrm{~h}$. The cells were then harvested by centrifugation and stored at $193 \mathrm{~K}$.

\subsection{Protein purification}

The cells were resuspended in lysis buffer $[500 \mathrm{mM} \mathrm{NaCl}$, $50 \mathrm{~m} M$ Tris pH 7.5, $30 \mathrm{~m} M$ imidazole, $0.2 \%(v / v)$ Tween], lysed

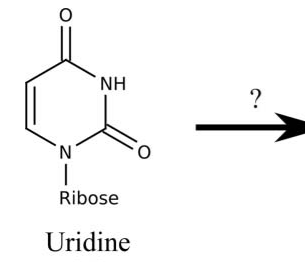

(U)<smiles>Cn1cc(O)c(=O)[nH]c1=O</smiles>

5-Hydroxyuridine $\left(h o^{5} \mathrm{U}\right)$

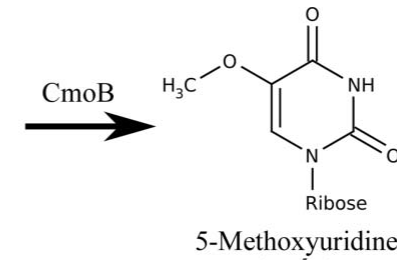

$\left(\mathrm{mo}^{5} \mathrm{U}\right)$

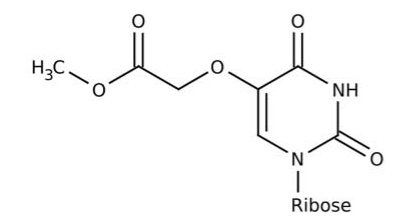

Uridine-5-oxyacetic acid methyl ester $\left(\mathrm{mcmo}^{5} \mathrm{U}\right)$
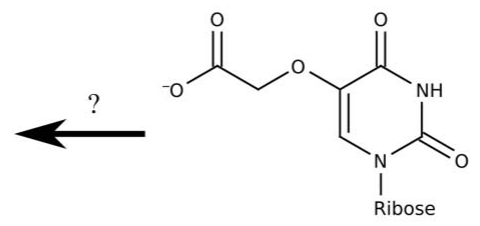

Uridine-5-oxyacetic acid $\left(\mathrm{cmo}^{5} \mathrm{U}\right)$
The proposed modification pathway of 5-oxyuridine derivatives. CmoA has been implicated in the modification of $\mathrm{mo}^{5} \mathrm{U}$ to $\mathrm{cmo}^{5} \mathrm{U}$ (Nasvall et al., 2004), although this reaction involves more than the addition of a single methyl group, indicating that either additional enzymes and/or cofactors are involved. No enzymes involved in the conversion of $\mathrm{U}$ to $\mathrm{ho}^{5} \mathrm{U}$ or $\mathrm{cmo}^{5} \mathrm{U}$ to $\mathrm{mcmo}^{5} \mathrm{U}$ have been 
using a Basic Z cell disruptor (Constant Systems) and clarified by centrifugation. The supernatant was loaded onto a $1 \mathrm{ml}$ HisTrap FF column (GE Healthcare) equilibrated with wash buffer (500 $\mathrm{m} M \mathrm{NaCl}, 50 \mathrm{~m} M$ Tris $\mathrm{pH}$ 7.5, $30 \mathrm{~m} M$ imidazole) and bound protein was eluted with elution buffer $(500 \mathrm{~m} M$ $\mathrm{NaCl}, 50 \mathrm{~m} M$ Tris pH 7.5, $500 \mathrm{~m} M$ imidazole). Fractions containing $\mathrm{CmoA}$ were concentrated and loaded onto a Superdex 200 HiLoad 16/60 column (GE Healthcare) equilibrated with gel-filtration buffer $(200 \mathrm{~m} M \mathrm{NaCl}, 20 \mathrm{~m} M$ Tris $\mathrm{pH}$ 7.5). Fractions containing $\mathrm{CmoA}$ were pooled and the $\mathrm{N}$-terminal hexahistidine tag was removed by digesting the protein with rhinovirus $3 \mathrm{C}$ protease. The mixture was then reverse-purified by performing an additional round of $\mathrm{Ni}^{2+}$ affinity chromatography as described above and collecting the flowthrough. This protein was then buffer-exchanged into gelfiltration buffer and concentrated to $20 \mathrm{mg} \mathrm{ml}^{-1}$ for crystallization.

\subsection{Size-exclusion chromatography coupled with static light scattering (SEC-SLS) analysis}

The oligomeric state of $\mathrm{CmoA}$ in solution was analysed using size-exclusion chromatography with a Superdex 200 column followed by light scattering using a Viscotek Tetra Array Detector measuring refractive index, right-angle light scattering and absorbance at $280 \mathrm{~nm}$. A $100 \mu \mathrm{l}$ sample at $0.77 \mathrm{mg} \mathrm{ml}^{-1}$ was applied onto the size-exclusion column and was observed to correspond to dimeric CmoA.

\subsection{Crystallization}

Sitting-drop experiments were performed in a CrystalQuick crystallization plate (Greiner Bio-One) at $294 \mathrm{~K} .100 \mathrm{nl} \mathrm{CmoA}$ solution was mixed with $100 \mathrm{nl}$ crystallization solution and equilibrated against a reservoir of $200 \mu \mathrm{l}$ crystallization solution. Crystals were grown in condition E8 of the Morpheus crystallization screen (Molecular Dimensions): $0.3 M$ diethylene glycol, $0.3 M$ triethylene glycol, $0.3 M$ tetraethylene glycol, 0.3 $M$ pentaethylene glycol, 0.1 $M$ MOPS/HEPES-Na $\mathrm{pH} 7.5,12.5 \%(w / v)$ PEG 1000, $12.5 \%(w / v)$ PEG 3350, $12.5 \%(w / v)$ MPD (Gorrec, 2009). Crystals grew after $5 \mathrm{~h}$ and were flash-cooled in liquid nitrogen without any additional cryoprotection.

\subsection{Crystallography}

Data were collected on beamline I04 of Diamond Light Source, Didcot, England and were processed with xia2 (Winter, 2010, Evans, 2011; Leslie, 2006; Sauter et al., 2004; Zhang et al., 2006). The structure was determined by molecular replacement using the structure of $H$. influenzae $\mathrm{YecO}$ (PDB entry 1im8; chain B; Lim et al., 2001) with both the SAM cofactor and solvent molecules removed and the programs CHAINSAW (Stein, 2008) and MOLREP (Vagin \& Teplyakov, 2010) as implemented in the MrBUMP pipeline (Keegan \& Winn, 2007). The molecular-replacement solution contained two molecules of CmoA and had initial $R_{\text {work }} / R_{\text {free }}$ values of $45.9 / 45.5 \%$. The model was then improved through alternate cycles of manual rebuilding using Coot (Emsley et al.,
Table 1

Data-collection and refinement statistics.

Values in parentheses are for the highest resolution shell.

\begin{tabular}{|c|c|}
\hline \multicolumn{2}{|l|}{ Data collection } \\
\hline Wavelength $(\AA)$ & 0.9795 \\
\hline Space group & $P 2_{1} 2_{1} 2$ \\
\hline Unit-cell parameters $(\AA)$ & $a=77.12, b=91.38, c=70.64$ \\
\hline Resolution $(\AA)$ & $55.9-1.73(1.78-1.73)$ \\
\hline \multicolumn{2}{|l|}{ No. of reflections } \\
\hline Total & 261435 (19274) \\
\hline Unique & $52750(3856)$ \\
\hline Completeness (\%) & $99.9(100.0)$ \\
\hline Multiplicity & $5.0(5.0)$ \\
\hline$\langle I / \sigma(I)\rangle$ & $15.1(2.0)$ \\
\hline$R_{\text {merge }}^{\dagger}$ & $0.056(0.648)$ \\
\hline$R_{\text {p.i.m } \neq} \ddagger$ & $0.034(0.366)$ \\
\hline Wilson $B$ factor $\left(\AA^{2}\right)$ & 21.6 \\
\hline \multicolumn{2}{|l|}{ Refinement } \\
\hline Resolution $(\AA)$ & $55.9-1.73(1.78-1.73)$ \\
\hline \multicolumn{2}{|l|}{ No. of reflections } \\
\hline Working & $50019(3640)$ \\
\hline Free & 2687 (212) \\
\hline \multicolumn{2}{|l|}{ No. of atoms } \\
\hline Total & 3942 \\
\hline Protein & 3593 \\
\hline SCM-SAH & 60 \\
\hline Solvent & 289 \\
\hline$R_{\text {work }} \S(\%)$ & $19.6(29.6)$ \\
\hline$R_{\text {free }}(\%)$ & $23.1(31.9)$ \\
\hline \multicolumn{2}{|l|}{ Mean $B$ factor $\left(\AA^{2}\right)$} \\
\hline Overall & 31.1 \\
\hline Protein & 30.7 \\
\hline SCM-SAH & 29.6 \\
\hline Solvent & 36.0 \\
\hline \multicolumn{2}{|l|}{ Geometry } \\
\hline R.m.s.d., bond lengths $(\AA)$ & 0.014 \\
\hline R.m.s.d., bond angles $\left({ }^{\circ}\right)$ & 1.7 \\
\hline \multicolumn{2}{|l|}{ Ramachandran plot (\%) } \\
\hline Favoured & 98.2 \\
\hline Allowed & 1.8 \\
\hline
\end{tabular}

$\dagger R_{\text {merge }}=\sum_{h k l} \sum_{i}\left|I_{i}(h k l)-\langle I(h k l)\rangle\right| / \sum_{h k l} \sum_{i} I_{i}(h k l) . \quad \ddagger R_{\text {p.i.m. }}$

$\sum_{h k l}[1 /[N(h k l)-1]\}^{1 / 2} \sum_{i}\left|I_{i}(h k l)-\langle I(h k l)\rangle\right| / \sum_{h k l} \sum_{i} I_{i}(h k l) . \quad \& R_{\text {work }}$ and $R_{\text {free }}=$ $\sum_{h k l}|| F_{\text {obs }}|-| F_{\text {calc }}|| / \sum_{h k l}\left|F_{\text {obs }}\right| . R_{\text {free }}$ was calculated from a randomly chosen set of reflections ( $5 \%$ of the total) excluded from the $R_{\text {work }}$ set used for refinement.

2010) and restrained refinement with REFMAC5 (Murshudov et al., 2011) using an isotropic $B$ factor for each atom and one TLS group per chain (Winn et al., 2001). A restraints file for $S$-adenosyl- $S$-carboxymethyl-L-homocysteine was created using the PRODRG2 server (Schüttelkopf \& van Aalten, 2004).

The final model contains two molecules of CmoA (residues 19-247 in chain $A$ and residues $20-244$ in chain $B$ ), two molecules of $S$-adenosyl-S-carboxymethyl-L-homocysteine, two molecules of MPD and 273 water molecules. Model statistics are provided in Table 1. The Ramachandran plot of the model was calculated with RAMPAGE (Lovell et al., 2003) and the figures were created with CCP4mg (McNicholas et al., 2011) and the PoseView server (Stierand et al., 2006). The coordinates and structure factors have been deposited in the Protein Data Bank with accession code 4iwn.

\subsection{Mass spectrometry}

Samples of CmoA were purified as described above and diluted to a concentration of $5 \mu M$ in $50 \%(v / v)$ aqueous 
acetonitrile containing $1 \%$ formic acid. These samples were introduced into the mass spectrometer using a TriVersa NanoMate ion source (Advion BioSciences) in positive-ion mode. Mass spectra were acquired using a solariX FT-MS (Bruker Daltonics) with a $9.4 \mathrm{~T}$ superconducting magnet. Tandem MS of the released ligand was performed by collisioninduced dissociation in the hexapole (Q-CID) with argon collision gas. Spectra were processed using DataAnalysis v.4.0 (Bruker Daltonics). Protein mass deconvolution was performed using v.2.0 of the SNAP algorithm and mass measurements from released ligand and fragmentation spectra were calculated from centroided data.

\subsection{Systematic name of the cofactor}

The IUPAC name for the $S$-adenosyl-S-carboxymethylL-homocysteine (SCM-SAH) cofactor is [(3S)-3-amino3-carboxypropyl $]\{[(2 S, 3 S, 4 R, 5 R)-5$-(6-aminopurin-9-yl)-3,4dihydroxyoxolan-2-yl]methyl\}(carboxymethyl)sulfanium.
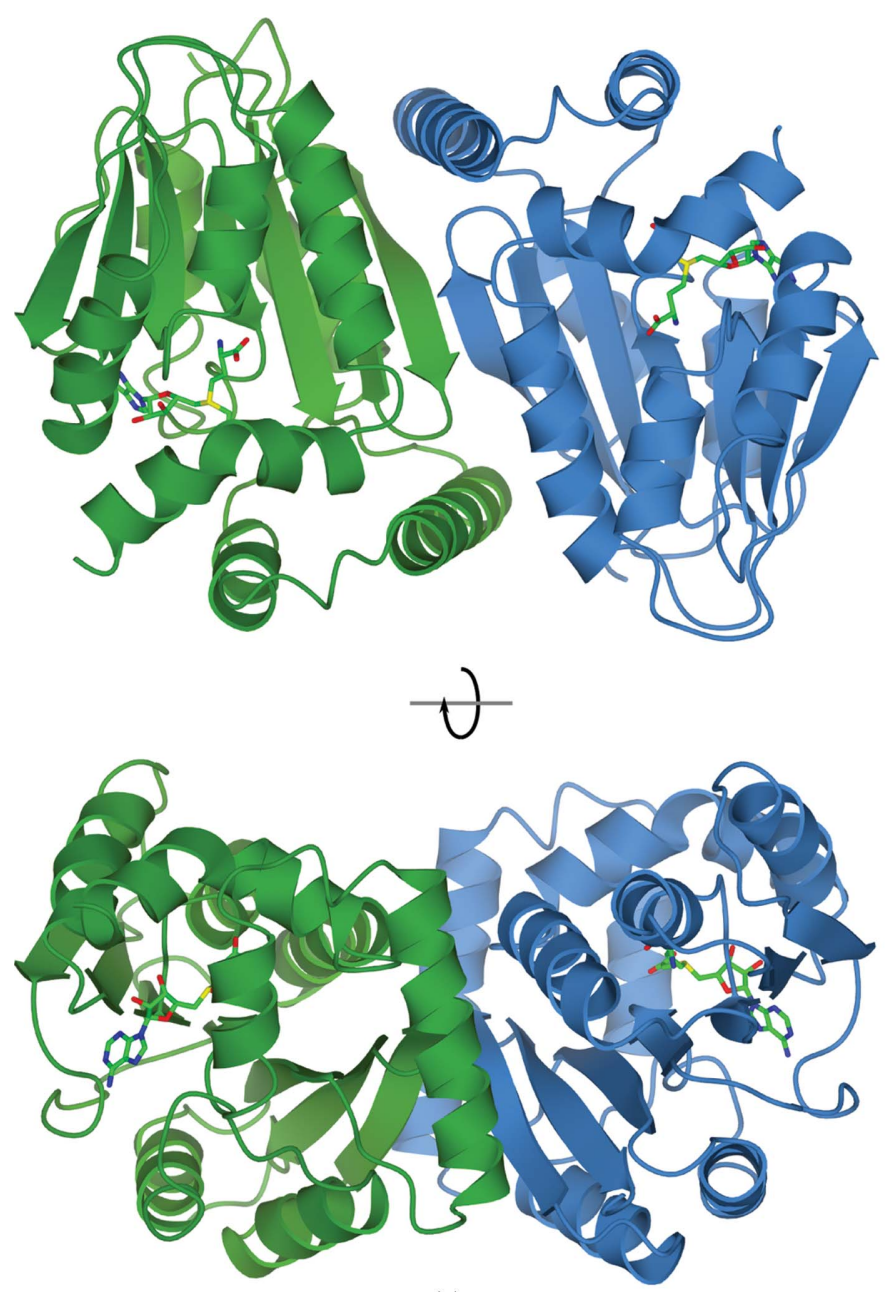

(a)

\section{Results and discussion}

The crystal structure of E. coli $\mathrm{CmoA}$ was determined by molecular replacement and was refined to $R_{\text {work }}$ and $R_{\text {free }}$ values of 19.6 and $23.1 \%$, respectively, using data to a resolution of $1.73 \AA$ (Table 1). The protein copurified with a cofactor from the E. coli cells that we anticipated would be either $S$-adenosylmethionine (SAM) or $S$-adenosylhomocysteine (SAH) on the basis of the $S$-adenosylmethionine-binding motifs present in the sequence of CmoA. Unexpectedly, both molecules of CmoA contain the novel derivative $S$-adenosyl$S$-carboxymethyl-L-homocysteine (SCM-SAH), which differs from SAM by the substitution of the methyl donor group $\left(R=-\mathrm{CH}_{3}\right)$ by a carboxymethyl group $\left(R=-\mathrm{CH}_{2} \mathrm{COOH}\right)$.

There are two molecules of CmoA present in the asymmetric unit that are related to each other by a noncrystallographic twofold rotational axis. Apart from minor differences at the $\mathrm{N}$ - and $\mathrm{C}$-termini, the two molecules adopt the same conformation and superpose with an r.m.s.d. of $0.3 \AA$ (225 aligned $\mathrm{C}^{\alpha}$ atoms). Analysis of the structure with PISA (Krissinel \& Henrick, 2007) reveals that the interface between the two molecules is extensive, with $1274 \AA^{2}$ of buried surface area per monomer (Fig. 2). In addition to an antiparallel $\beta$-sheet formed by the $\beta 6$ strands of both molecules, there are additional interactions between helix $\alpha 6$ of one molecule and helix $\alpha 2$ and strand $\beta 6$ of the other molecule. Together, these interactions comprise 15 hydrogen bonds and a number of hydrophobic interactions, suggesting that the CmoA dimer present in the asymmetric unit may also represent the oligomeric state of CmoA in solution. To confirm the existence of this dimer in solution, purified CmoA was analysed by sizeexclusion chromatography and static light scattering (SECSLS). A single species was visible on the chromatogram and

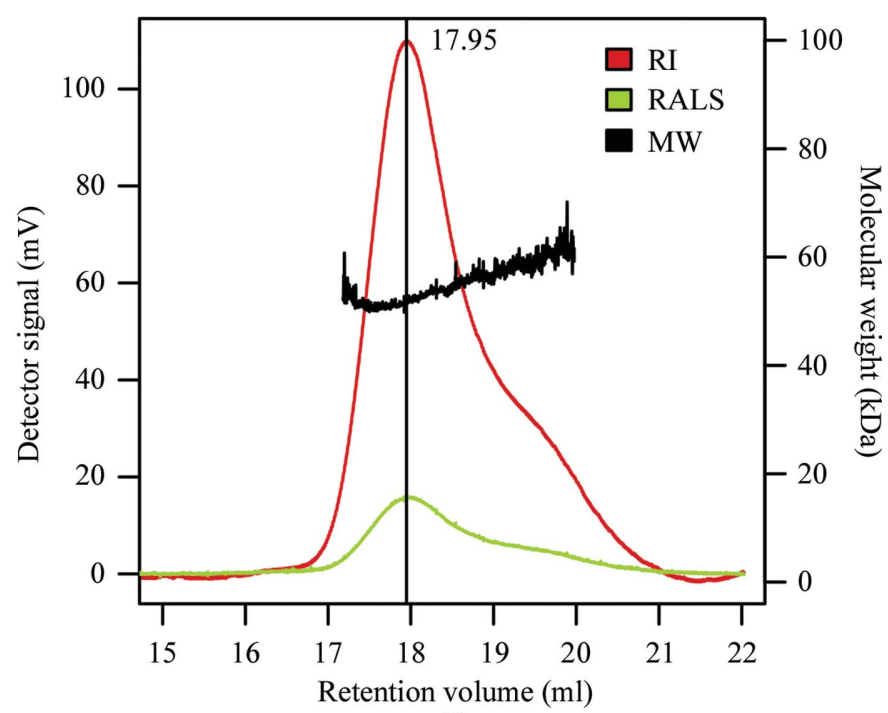

(b)

Figure 2

The overall structure of the CmoA dimer. (a) Two orthogonal views of the two monomers forming the dimer (green and blue ribbons). The $S$-adenosyl- $S$ carboxymethyl-L-homocysteine (SCM-SAH) cofactor is shown with $\mathrm{O}$ atoms in red, $\mathrm{N}$ atoms in blue and $\mathrm{C}$ atoms in green. $(b)$ The SEC-SLS chromatogram that confirms that $\mathrm{CmoA}$ is a dimer in solution. The refractive index (RI; red line) and right-angle light scattering (RALS; green line) traces are displayed. The molecular weight calculated by the OmniSEC software (MW; black line) is shown above the elution peak. The dispersity, $\mathrm{Mw} / \mathrm{Mn}$, defined as the ratio of the weight average to number average molecular weights was reported to be 1.001 , indicating a highly uniform sample. 


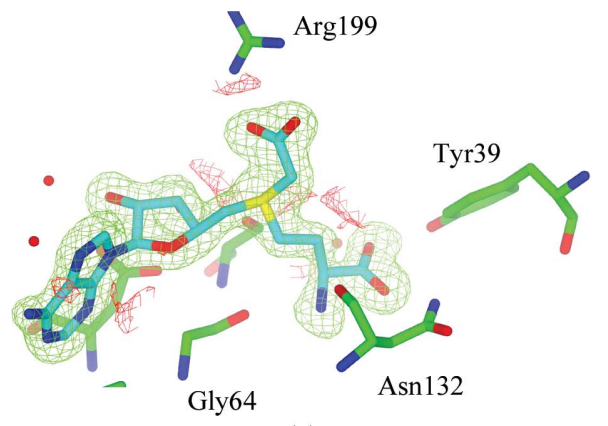

(a)

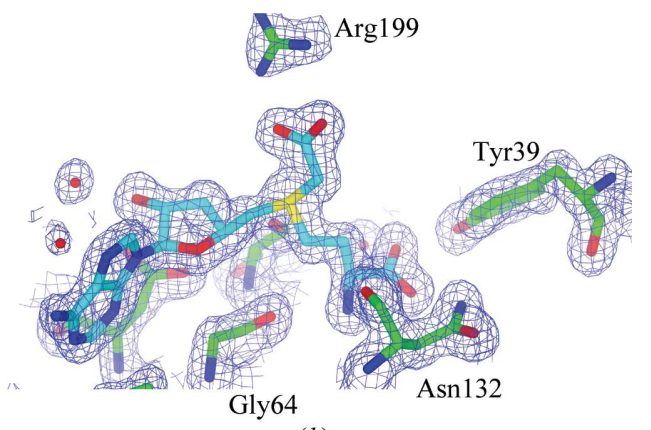

(b)

Figure 3

Structure of $S$-adenosyl-S-carboxymethyl-L-homocysteine (SCM-SAH). The final coordinates are displayed with $(a)$ the likelihood-weighted $m F_{\mathrm{o}}-D F_{\mathrm{c}}$ difference electron-density maps contoured at $3 \sigma$ calculated prior to the modelling of SCM-SAH and $(b)$ the $2 m F_{\mathrm{o}}-D F_{\mathrm{c}}$ electron-density maps contoured at $1.5 \sigma$. The SCM-SAH model is shown with $\mathrm{C}$ atoms in cyan, $\mathrm{O}$ atoms in red and $\mathrm{N}$ atoms in blue. $\mathrm{C}$ atoms of protein residues are shown in green.

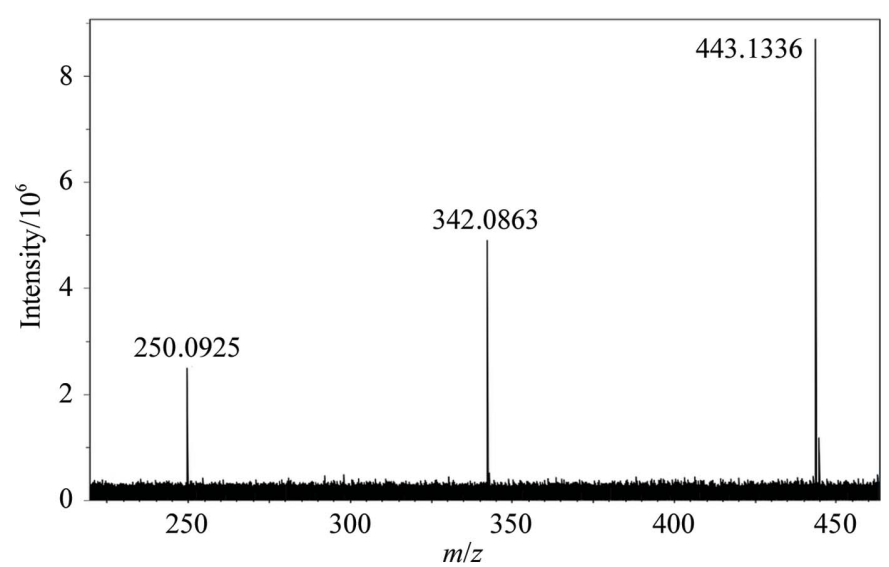

(a)

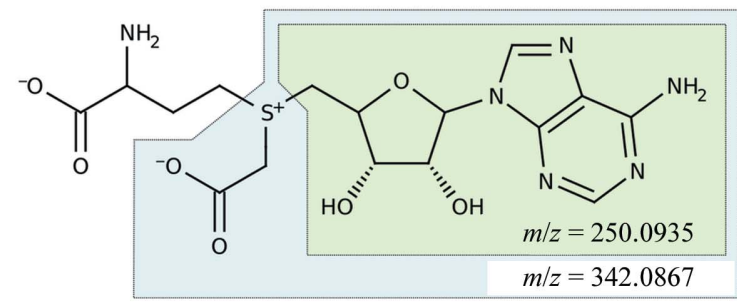

(b)

\section{Figure 4}

Mass spectrum of SCM-SAH. (a) The fragmentation spectrum and (b) the chemical structure of SCM-SAH. Signals consistent with the entire SCM-SAH cofactor as well as two fragments (in the blue and green boxes) were detected.

the molecular weight was calculated to be $52.5 \mathrm{kDa}$, which is consistent with the theoretical molecular weight of $55.6 \mathrm{kDa}$ for the dimer (Fig. 2). Interestingly, PISA identifies an equivalent dimer in the crystal structure of $H$. influenzae $\mathrm{YecO}$ (PDB entry 1im8) between chain $A$ and its symmetry mate $(x-y,-y,-z)$ with an interface area of $1194 \AA^{2}$ per monomer (Lim et al., 2001). The conserved nature of this interface indicates that the oligomeric state may be important for the structure and function of CmoA.

\subsection{CmoA contains a novel $S$-adenosylmethionine derivative}

During the refinement of the structure, it became apparent from inspection of both the $2 m F_{\mathrm{o}}-D F_{\mathrm{c}}$ and the $m F_{\mathrm{o}}-D F_{\mathrm{c}}$

electron-density maps that the putative active site of CmoA contained a cofactor that was neither $S$-adenosylmethionine (SAM) nor $S$-adenosylhomocysteine (SAH). Prior to the modelling of the ligand, unambiguous positive density was visible in the $m F_{\mathrm{o}}-D F_{\mathrm{c}}$ electron-density map for all of the features expected for SAM: L-methionine and both the adenine and ribose rings were visible at a contour level of $5 \sigma$, while the $\mathrm{S}$ atom was visible at a contour level of $18 \sigma$ (Fig. 3a). However, additional positive density was present at the end of the methyl group, indicating that the cofactor was a covalently modified derivative of SAM. This positive density was bifurcated, planar in shape and visible at a contour level of $7 \sigma$. Because these maps were generated prior to the modelling of any cofactor and no cofactor was present in the search model that was used during molecular replacement, the presence of this additional density was not a consequence of model bias. The shape of the density was most consistent with an $S$-adenosylmethionine derivative in which the methyl group had been derivatized with a functional group with trigonal planar geometry.

To confirm the presence of the cofactor and investigate its identity, samples of CmoA were analysed by Fourier transform mass spectrometry (FT-MS). To exclude the possibility that the modification occurred during crystallization or data collection (as a result of the chemicals present in the crystallization solution or of exposure to X-rays), the sample used for analysis was not crystallized but was from the same preparation as that used for crystallization. A signal at $m / z=27764.2$ was assigned as a protonated molecular-ion peak for CmoA, in agreement with the value of $\mathrm{m} / z=27763.9$ estimated from the sequence alone (a difference of $0.3 \mathrm{Da}$ after accounting for the proton). An additional signal at $m / z=443.1333$ was detected and this was isolated and further analysed by collision-induced dissociation. The resulting fragmentation spectrum contained three signals: a parent ion at $\mathrm{m} / \mathrm{z}=443.1336$ and two fragments at $m / z=342.0863$ and 250.0925 (Fig. 4a). These are not consistent with the theoretical values for SAM $(m / z=399.1445)$ or SAH $(m / z=385.1289)$, and the fragmentation spectrum featured no signals at these values. A search of the PubChem database for compounds structurally similar to SAH or SAM with a molecular weight of between 442.6 and 443.6 Da resulted in single hit: a SAM derivative in which the methyl group is substituted by a carboxymethyl group (CID 11212932; Fig. 4b). We refer to this derivative as $S$-adenosyl- $S$-carboxymethyl-L-homocysteine (or $S$-carboxymethylated SAH), which could be further abbreviated as SCM-SAH; the full IUPAC systematic name is given in $\$ 2.7$. The $\mathrm{m} / \mathrm{z}$ values calculated for this compound are in close agreement with those determined experimentally: the parent 
ion has a calculated value of $\mathrm{m} / \mathrm{z}=443.1336(-0.70 \mathrm{mDa}$ difference) and two potential fragments may be generated with calculated values of $\mathrm{m} / z=342.0867$ and $250.0935(-0.33$ and $-0.93 \mathrm{mDa}$ difference, respectively; Figs. $4 a$ and $4 b$ ).

Restraints for the modelling and refinement of SCM-SAH were generated with PRODRG2 and the ligand was then modelled into the active site of each monomer (Fig. $3 b$ ). Following refinement, there was no obvious distortion of the ligand geometry with respect to the ideal geometry and there were no significant positive or negative peaks in the $m F_{\mathrm{o}}-D F_{\mathrm{c}}$ difference electron-density map in the immediate vicinity of the ligand. Taken together, the crystallographic and massspectrometric data suggest that the active site of $\mathrm{CmoA}$ contains an $S$-adenosylmethionine derivative in which the methyl group is substituted by a carboxymethyl group.

\subsection{Comparison with $\boldsymbol{H}$. influenzae YecO}

H. influenzae YecO (PDB entry 1im8) was identified as the most similar deposited structure to $\mathrm{CmoA}$ by both sequencebased ( $68 \%$ sequence identity) and structure-based (r.m.s.d. = $0.58 \AA$ for 222 aligned $\mathrm{C}^{\alpha}$ atoms) search methods (Krissinel \& Henrick, 2004). The structure of $\mathrm{YecO}$ was originally determined by multiple-wavelength anomalous diffraction (MAD) using a selenomethionine derivative produced in E. coli B834 (DE3) cells grown in minimal medium in the presence of L-selenomethionine (Lim et al., 2001). The authors noted that in addition to the number of selenium sites expected on the basis of the protein sequence, one additional selenium site per monomer was detected during structure determination. This was incorporated into the cofactor that copurified with $\mathrm{YecO}$, suggesting that the cofactor was derived from L-methionine (in the case of cells grown in non-labelled medium) or L-selenomethionine (in the case of cells grown in minimal medium with L-selenomethionine). In the deposited structure of $\mathrm{YecO}$ the cofactor was modelled as Se-substituted $\mathrm{SAH}$ with a $\mathrm{Cl}^{-}$ion $2.9 \AA$ away from the Se atom.

Given the high degree of similarity between CmoA and $\mathrm{YecO}$, we re-examined both the coordinates and the structure factors for YecO deposited in the PDB. Re-refinement of the deposited structure resulted in $R_{\text {work }}$ and $R_{\text {free }}$ values of 19.2 and $24.8 \%$, respectively, which are comparable with the values of 18.6 and $25.5 \%$ originally reported (Fig. $5 a$ ). Refinement of the $\mathrm{YecO}$ structure without any cofactor modelled results in electron density in both the $2 m F_{\mathrm{o}}-D F_{\mathrm{c}}$ and the $m F_{\mathrm{o}}-D F_{\mathrm{c}}$ electron-density maps into which the Se-substituted form of the SCM-SAH cofactor found in CmoA can be modelled, and the electron-density maps after refinement are also compatible with the presence of this cofactor (Fig. 5b). However, the electron density is not defined well enough to allow a distinction between the possibilities of SAH and a $\mathrm{Cl}^{-}$ion (as modelled originally) or of SCM-SAH (as modelled in CmoA). We note, however, that we were unable to find evidence of $\mathrm{SAH}$ and an equivalently positioned $\mathrm{Cl}^{-}$ion in a manual inspection of PDB entries that are (i) annotated as methyltransferases (EC 2.1.1) and (ii) contain at least one $\mathrm{Cl}^{-}$ion. Furthermore, in the case of $\mathrm{CmoA}$ the electron-density maps are better defined and the mass-spectrometric data argue against the cofactor modelled in YecO.

\subsection{Overall structure of $\mathrm{CmoA}$}

CmoA has a Rossmann fold that comprises seven $\beta$-strands and eight $\alpha$-helices. The $\beta$-strands form a single sheet in which all strands are parallel except $\beta 7$. The majority of the $\alpha$-helices pack against both faces of the $\beta$-sheet, although helices $\alpha 2, \alpha 6$ and $\alpha 7$ form a compact lid-like structure that sits over the region containing the SCM-SAH and renders it almost inaccessible to solvent. Superposition of $\mathrm{CmoA}$ with the structures of the other RNA methyltransferases TrmA (PBD entry 3bt7) and RumA (RlmD; PDB entry 2bh2) reveals that while the Rossmann-fold core is conserved between these enzymes, the lid-like structure of $\mathrm{CmoA}$ obstructs the region used for the binding of the RNA substrate in these methyltransferases (Lee et al., 2005; Alian et al., 2008). The conserved location of the substrate nucleoside with respect to the SAM cofactor in $\operatorname{TrmA}$, RumA and other DNA and RNA methyltransferases
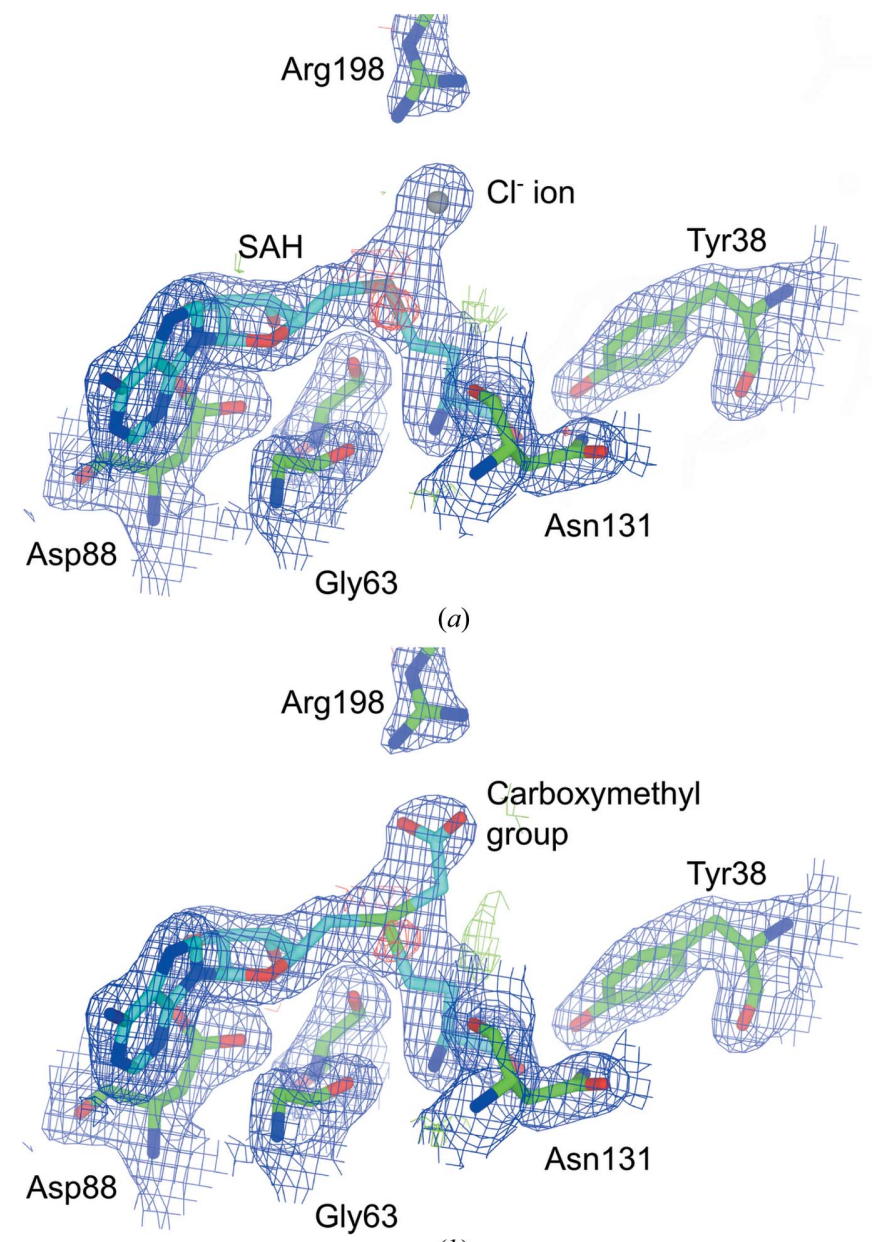

(b)

Figure 5

Modelling of SCM-SAH into YecO. The re-refined coordinates with either $(a)$ the originally modelled Se-substituted SAH and $\mathrm{Cl}^{-}$ion or $(b)$ remodelled SCM-SAH are displayed along with the $2 m F_{\mathrm{o}}-D F_{\mathrm{c}}$ electron-density maps contoured at $1 \sigma$ and the $m F_{\mathrm{o}}-D F_{\mathrm{c}}$ difference electron-density maps contoured at $3 \sigma$. SCM-SAH is depicted with $\mathrm{C}$ atoms in cyan, while $\mathrm{C}$ atoms of protein residues are shown in green. 
suggests that the binding mode is relatively fixed. In order to place the substrate nucleoside in the corresponding position with respect to the SCM-SAH cofactor in CmoA, the lid would need to undergo a large conformational change to allow access to the cofactor and to prevent significant clashes with the neighbouring nucleotides of the tRNA substrate. An alternative possibility is that $\mathrm{CmoA}$ acts using additional factors which assist in the modification of the substrate uridine and does not bind tRNA directly.

In common with many enzymes possessing a Rossmann fold, the majority of the conserved residues are located at the C-terminal ends of the $\beta$-strands or in the loops which

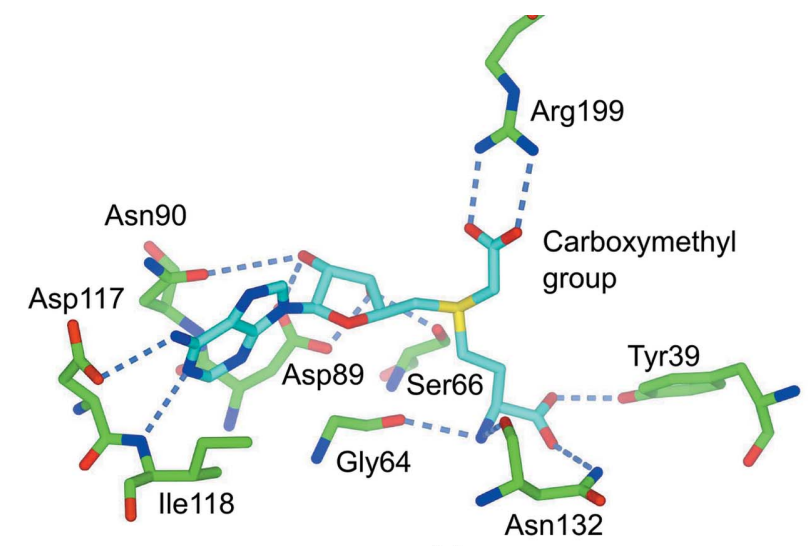

(a)

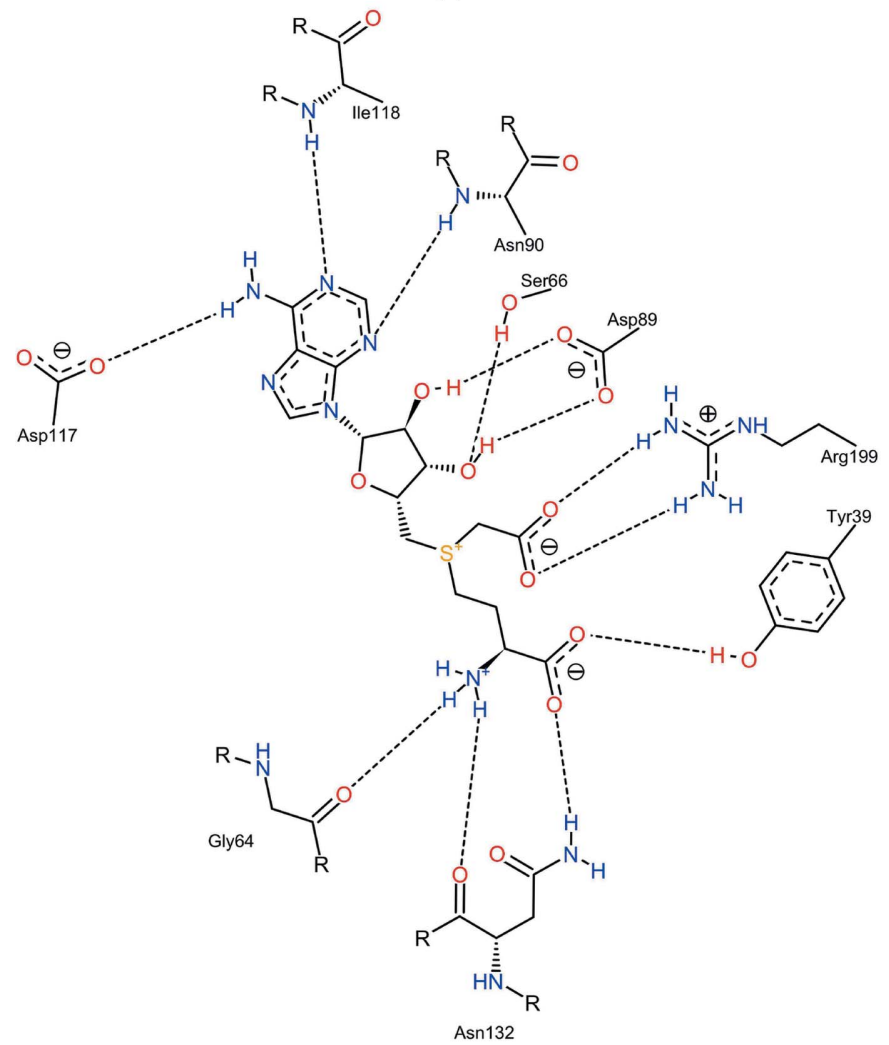

(b)

Figure 6

Binding of SCM-SAH by CmoA. (a) SCM-SAH (cyan cylinders) interacts with multiple main-chain and side-chain atoms of $\mathrm{CmoA}$ (green cylinders) through hydrogen bonds (blue dashes). (b) A two-dimensional schematic of the active site. immediately follow. In $\mathrm{CmoA}$ these residues are directly involved in contacting the SAM derivative: the adenine ring is hydrogen-bonded by the side chain of Asp117 and the main chains of Asn90, Ile118 and Phe137 (via a water molecule), the ribose is hydrogen bonded by the side chains of Ser66, Asp89 and Asn90 (via a water molecule), and the L-methionine is hydrogen bonded by the side chains of Tyr39, Asp62 (via a water molecule) and Asn132 and the main chains of Gly64, Ala70 (via a water molecule) and Asn132 (Figs. 6a and 6b). The negatively charged carboxylate of the carboxymethyl group interacts with the positively charged guanidinium group of Arg199 through a salt bridge with a length of $2.7 \AA$. The high conservation of these residues within members of the UniProt CmoA family indicates that they are important for binding the SAM derivative and may also play a role in its biochemistry.

\section{Conclusions}

We have determined the structure of E. coli CmoA, a putative methyltransferase that is involved in the post-transcriptional modification of U34 in a number of bacterial tRNAs. While the sequence motifs and Rossmann fold of the enzyme suggest that it is a typical SAM-dependent methyltransferase, analysis of the electron-density maps and mass-spectrometric data revealed that the protein contains an atypical SAM derivative in which the donor methyl group is replaced by a carboxymethyl group. We name this previously unobserved derivative $S$-adenosyl-S-carboxymethyl-L-homocysteine (SCM-SAH). According to the UniProt database, the $\mathrm{CmoA}$ family currently contains 1566 proteins that are currently annotated as putative SAM-dependent methyltransferases. However, conservation of $\operatorname{Arg} 199$, the key residue of CmoA that stabilizes the negative charge of the carboxyl group of the SCM-SAH cofactor, suggests that these proteins contain the SCM-SAH cofactor instead of SAM and are currently annotated incorrectly. The equivalent residue in known SAMdependent methyltransferases is not conserved. Although CmoA homologues are only found in bacteria, it is possible that such SAM derivatives are widespread in nature, being present in other enzymes currently annotated as methyltransferases.

Previous genetic studies have indicated that $\mathrm{CmoA}$ is involved in the modification of $\mathrm{mo}^{5} \mathrm{U}$ to $\mathrm{cmo}^{5} \mathrm{U}$, a reaction that involves the addition of a carboxyl group onto the methoxy group of $\mathrm{mo}^{5} \mathrm{U}$ but that cannot be catalysed solely by a methyltransferase (Nasvall et al., 2004). Assuming that the cofactor in $\mathrm{CmoA}$ is directly involved in modification of the tRNA, we speculate that it may participate in the formation of $\mathrm{cmo}^{5} \mathrm{U}$ by either (i) the transfer of just the carboxyl group of SCM-SAH onto the methoxy group of $\mathrm{mo}^{5} \mathrm{U}$ or (ii) the substitution of the methyl group of the side chain in $\mathrm{mo}^{5} \mathrm{U}$ by the entire carboxymethyl group from SCM-SAH (Fig. 7). A third possibility is that the carboxymethyl group is transferred directly onto the hydroxyl group of ho ${ }^{5} \mathrm{U}$. Although this proposal is not supported by the observation that mutations in cmo $A$ result in accumulation of $\mathrm{mo}^{5} \mathrm{U}$ and not $\mathrm{ho}^{5} \mathrm{U}$, this has 


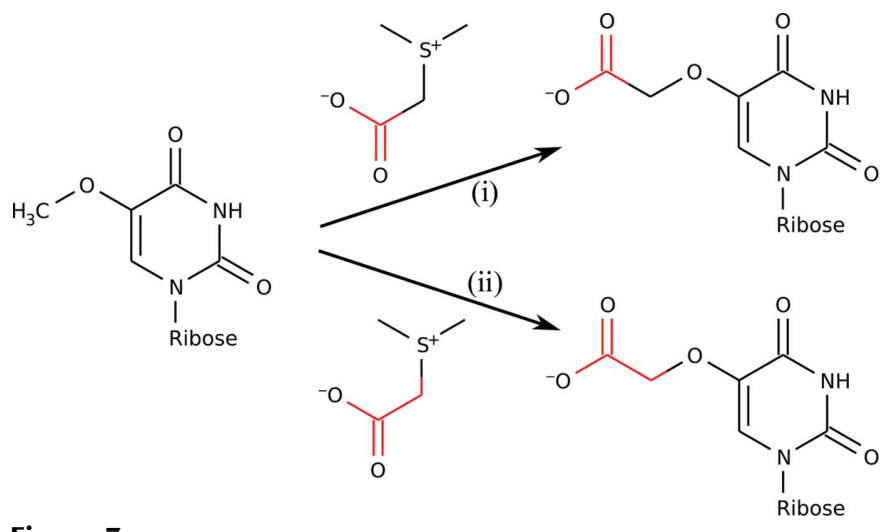

Figure 7

Speculative roles for SCM-SAH in the modification of $\mathrm{mo}^{5} \mathrm{U}$. The modification might involve either (i) the addition of the carboxyl group (red) from SCM-SAH onto the methoxy group of $\mathrm{mo}^{5} \mathrm{U}$ or (ii) the substitution of the methyl group of $\mathrm{mo}^{5} \mathrm{U}$ with the entire carboxymethyl group.

been suggested previously (Murao et al., 1978) and a precedent for the chemistry of this reaction can be found in the O-methyltransferases. All three possibilities, however, would be compatible with the observation that only one of the $\mathrm{C}$ atoms in the side chain of $\mathrm{cmo}^{5} \mathrm{U}$ is derived from L-methionine. The proposed mechanisms for SAM-dependent methyltransferases often involve a general base. Superpositions of $\mathrm{CmoA}$ with the 5-methyluridine methyltransferases TrmA and RumA show that the C5 atom of the substrate uridine is neighboured by Glu164. This residue is highly conserved in members of the CmoA family and the only other amino acid found at this position is aspartic acid. As the side chain of $\mathrm{cmo}^{5} \mathrm{U}$ derivatives is attached to the $\mathrm{C} 5$ atom of the pyrimidine ring, this residue would potentially be able to act as a general base during the reaction.

Although DNA and RNA methyltransferases are able to use synthetic $S$-adenosylmethionine analogues with extended carbon chains both in vitro and in vivo (Schlenk \& Dainko, 1975; Klimasauskas \& Weinhold, 2007; Motorin et al., 2011), there do not appear to be any reports indicating that DNA or RNA methyltransferases actually use such analogues in vivo for the modification of nucleic acids. We hope that our findings will lead to further characterization of the function and mechanism of CmoA and its SCM-SAH cofactor.

During the final stages of preparation of our manuscript, we became aware of PDB deposition 4gek by the New York Structural Genomics Research Consortium, in which E. coli CmoA is also observed in complex with SCM-SAH. A comparison of these independently determined structures adds support to the conclusions presented in this paper.

The authors would like to thank Gideon Grogan (York Structural Biology Laboratory, University of York, England) for useful suggestions. We would also like to thank Osnat Herzberg (Institute for Bioscience and Biotechnology Research, University of Maryland, USA) for a discussion regarding the structure of YecO. The OPPF-UK is funded by the Medical Research Council and the Biotechnology and
Biological Sciences Research Council. Wellcome Trust Centre for Human Genetics is supported by the Wellcome Trust (grant No. 075491). Mass-spectrometric experiments were funded by the Wellcome Trust grant 098230 to AAA. These experiments were performed at York Centre of Excellence in Mass Spectrometry funded by the Yorkshire Forward/ Northern Way Initiative. We thank Matthew Jennions from the Membrane Protein Laboratory, Imperial College for the SEC-SLS experiment and for assistance with interpreting the data.

\section{References}

Agris, P. F. (2008). EMBO Rep. 9, 629-635.

Agris, P. F., Vendeix, F. A. \& Graham, W. D. (2007). J. Mol. Biol. 366, $1-13$.

Alian, A., Lee, T. T., Griner, S. L., Stroud, R. M. \& Finer-Moore, J. (2008). Proc. Natl Acad. Sci. USA, 105, 6876-6881.

Berrow, N. S., Alderton, D., Sainsbury, S., Nettleship, J., Assenberg, R., Rahman, N., Stuart, D. I. \& Owens, R. J. (2007). Nucleic Acids Res. 35, e45.

Cantara, W. A., Crain, P. F., Rozenski, J., McCloskey, J. A., Harris, K. A., Zhang, X., Vendeix, F. A., Fabris, D. \& Agris, P. F. (2011). Nucleic Acids Res. 39, D195-D201.

Emsley, P., Lohkamp, B., Scott, W. G. \& Cowtan, K. (2010). Acta Cryst. D66, 486-501.

Evans, P. R. (2011). Acta Cryst. D67, 282-292.

Gorrec, F. (2009). J. Appl. Cryst. 42, 1035-1042.

Hagervall, T. G., Jönsson, Y. H., Edmonds, C. G., McCloskey, J. A. \& Björk, G. R. (1990). J. Bacteriol. 172, 252-259.

Jühling, F., Mörl, M., Hartmann, R. K., Sprinzl, M., Stadler, P. F. \& Pütz, J. (2009). Nucleic Acids Res. 37, D159-D162.

Keegan, R. M. \& Winn, M. D. (2007). Acta Cryst. D63, 447-457.

Klimasauskas, S. \& Weinhold, E. (2007). Trends Biotechnol. 25, 99-104.

Krissinel, E. \& Henrick, K. (2004). Acta Cryst. D60, 2256-2268.

Krissinel, E. \& Henrick, K. (2007). J. Mol. Biol. 372, 774-797.

Lee, T. T., Agarwalla, S. \& Stroud, R. M. (2005). Cell, 120, 599-611.

Leslie, A. G. W. (2006). Acta Cryst. D62, 48-57.

Lim, K., Zhang, H., Tempczyk, A., Bonander, N., Toedt, J., Howard, A., Eisenstein, E. \& Herzberg, O. (2001). Proteins, 45, 397-407.

Lovell, S. C., Davis, I. W., Arendall, W. B., de Bakker, P. I., Word, J. M., Prisant, M. G., Richardson, J. S. \& Richardson, D. C. (2003). Proteins, 50, 437-450.

McNicholas, S., Potterton, E., Wilson, K. S. \& Noble, M. E. M. (2011). Acta Cryst. D67, 386-394.

Motorin, Y., Burhenne, J., Teimer, R., Koynov, K., Willnow, S., Weinhold, E. \& Helm, M. (2011). Nucleic Acids Res. 39, 19431952.

Motorin, Y. \& Helm, M. (2010). Biochemistry, 49, 4934-4944.

Murao, K., Ishikura, H., Albani, M. \& Kersten, H. (1978). Nucleic Acids Res. 5, 1273-1281.

Murshudov, G. N., Skubák, P., Lebedev, A. A., Pannu, N. S., Steiner, R. A., Nicholls, R. A., Winn, M. D., Long, F. \& Vagin, A. A. (2011). Acta Cryst. D67, 355-367.

Nasvall, S. J., Chen, P. \& Bjork, G. R. (2004). RNA, 10, 1662-1673.

Sauter, N. K., Grosse-Kunstleve, R. W. \& Adams, P. D. (2004). J. Appl. Cryst. 37, 399-409.

Schlenk, F. \& Dainko, J. L. (1975). Biochim. Biophys. Acta, 385, 312-323.

Schüttelkopf, A. W. \& van Aalten, D. M. F. (2004). Acta Cryst. D60, 1355-1363.

Selmer, M., Dunham, C. M., Murphy, F. V. IV, Weixlbaumer, A., Petry, S., Kelley, A. C., Weir, J. R. \& Ramakrishnan, V. (2006). Science, 313, 1935-1942.

Shi, H. \& Moore, P. B. (2000). RNA, 6, 1091-1105. 


\section{research papers}

Stein, N. (2008). J. Appl. Cryst. 41, 641-643.

Stierand, K., Maass, P. C. \& Rarey, M. (2006). Bioinformatics, 22, 1710-1716.

Vagin, A. \& Teplyakov, A. (2010). Acta Cryst. D66, 22-25.

Vendeix, F. A., Dziergowska, A., Gustilo, E. M., Graham, W. D., Sproat, B., Malkiewicz, A. \& Agris, P. F. (2008). Biochemistry, 47,
6117-6129.

Winn, M. D., Isupov, M. N. \& Murshudov, G. N. (2001). Acta Cryst. D57, 122-133.

Winter, G. (2010). J. Appl. Cryst. 43, 186-190.

Zhang, Z., Sauter, N. K., van den Bedem, H., Snell, G. \& Deacon, A. M. (2006). J. Appl. Cryst. 39, 112-119. 